\title{
ON THE NON-VANISHING OF THE JACOBIAN IN CERTAIN ONE-TO-ONE MAPPINGS
}

\section{BY HANS LEWY}

THEOREM 1. If $u(x, y)$ and $v(x, y)$ are harmonic, $u(0,0)$ $=v(0,0)=0$, and if there exists a neighborhood $N_{1}$ of the origin of the xy plane and a neighborhood $N_{2}$ of the origin of the uv plane such that $u(x, y)$ and $v(x, y)$ establish a mapping of $N_{1}$ onto $N_{2}$ which is one-to-one both ways, then the Jacobian $\partial(u, v) / \partial(x, y)$ does not vanish at the origin.

Proof. As the statement of Theorem 1 remains invariant under homogeneous linear transformations of the $u v$ plane, we may assume, in the developments in polar coordinates for $u$ and $v$, that

$$
\begin{array}{ll}
u=\sum_{i}^{\infty}\left[a_{n} r^{n} \cos n \theta+b_{n} r^{n} \sin n \theta\right], & \left(a_{i}{ }^{2}+b_{i}{ }^{2} \neq 0\right), \\
v=\sum_{k}^{\infty}\left[A_{n} r^{n} \cos n \theta+B_{n} r^{n} \sin n \theta\right], & \left(A_{k}{ }^{2}+B_{k}{ }^{2} \neq 0\right),
\end{array}
$$

that the positive index $i$ does not exceed $k$, and that for $i=k$ we have $a_{k} B_{k}-A_{k} b_{k} \neq 0$. Considering the case $i=k$ first, we may, because of the invariance mentioned, assume

$$
a_{k}=B_{k}=1, \quad b_{k}=A_{k}=0 .
$$

For small values of $r$, the auxiliary mapping,

$$
\bar{u}=r^{k} \cos k \theta, \quad \bar{v}=r^{k} \sin k \theta,
$$

can be continuously joined with the given one for $0 \leqq t \leqq 1$ by

$$
\begin{aligned}
& u_{t}=r^{k} \cos k \theta+t \sum_{k+1}^{\infty}\left(a_{n} r^{n} \cos n \theta+b_{n} r^{n} \sin n \theta\right), \\
& v_{t}=r^{k} \sin k \theta+t \sum_{k+1}^{\infty}\left(A_{n} r^{n} \cos n \theta+B_{n} r^{n} \sin n \theta\right) .
\end{aligned}
$$

The vector $\left(u_{t}, v_{t}\right)$ thereby never differs by more than a vector of length $r^{k} / 2$ from the vector $(\bar{u}, \bar{v})$ whose length is $r^{k}$. Thus 
the index of the origin* in both fields $(u, v)$ and $(\bar{u}, \bar{v})$ is the same, and as it is \pm 1 for one-to-one mappings we conclude $i=k=1$ and our theorem follows.

In the remaining case $i<k$ we consider the Jacobian

$$
J=\frac{\partial(u, v)}{\partial(x, y)}=\frac{1}{r} \frac{\partial(u, v)}{\partial(r, \theta)} .
$$

We find by elementary computation that the development of $J$ starts with the terms of lowest degree in $r$

$k i r^{i+k-1}\left[\left(A_{k} a_{i}+B_{k} b_{i}\right) \sin (i-k) \theta+\left(a_{i} B_{k}-b_{i} A_{k}\right) \cos (i-k) \theta\right]$

or, with a suitable angle $\theta_{0}$, with the term

$$
k i r^{i+k-1}\left(A_{k}{ }^{2}+B_{k}{ }^{2}\right)^{1 / 2}\left(a_{i}{ }^{2}+b_{i}{ }^{2}\right)^{1 / 2} \cos \left[(i-k) \theta-\theta_{0}\right] .
$$

Hence, for sufficiently small values of $r, J$ assumes both positive and negative values, while in a one-to-one map the Jacobian, where it does not vanish, is of the same sign as the index of the map which is either +1 everywhere or -1 everywhere. Hence $i<k$ is impossible and our proof is completed.

TheOREM 2. Let $u(x, y)$ and $v(x, y)$ be analytic functions of $x$ and $y$ in a neighborhood $N_{1}$ of the origin of the xy plane which they map onto a neighborhood $N_{2}$ of the origin of the uv plane in a one-to-one correspondence. Suppose, moreover, that $u$ and $v$ are solutions of the following equations:

$$
\begin{aligned}
& \frac{\partial^{2} u}{\partial x^{2}}+\frac{\partial^{2} u}{\partial y^{2}}+a\left[\left(\frac{\partial u}{\partial x}\right)^{2}+\left(\frac{\partial u}{\partial y}\right)^{2}\right]+b\left(\frac{\partial u}{\partial x} \frac{\partial v}{\partial x}+\frac{\partial u}{\partial y} \frac{\partial v}{\partial y}\right) \\
&+c\left[\left(\frac{\partial v}{\partial x}\right)^{2}+\left(\frac{\partial v}{\partial y}\right)^{2}\right]+d\left(\frac{\partial u}{\partial x} \frac{\partial v}{\partial y}-\frac{\partial u}{\partial y} \frac{\partial v}{\partial x}\right)=0 \\
& \frac{\partial^{2} v}{\partial x^{2}}+\frac{\partial^{2} v}{\partial y^{2}}+A\left[\left(\frac{\partial u}{\partial x}\right)^{2}+\left(\frac{\partial u}{\partial y}\right)^{2}\right]+B\left(\frac{\partial u}{\partial x} \frac{\partial v}{\partial x}+\frac{\partial u}{\partial y} \frac{\partial v}{\partial y}\right) \\
&+C\left[\left(\frac{\partial v}{\partial x}\right)^{2}+\left(\frac{\partial v}{\partial y}\right)^{2}\right]+D\left(\frac{\partial u}{\partial x} \frac{\partial v}{\partial y}-\frac{\partial u}{\partial y} \frac{\partial v}{\partial x}\right)=0
\end{aligned}
$$

* For the topological notions used, see W. Fenchel, Elementare Berveise und Anwendungen einiger Fixpunktsätze, Matematisk Tidsskrift, (B), 1932, p. 66. 
in which $a(u, v), b(u, v), \cdots, D(u, v)$ are analytic functions of $u$ and $v$ defined for $(u, v)$ in $N_{2}$. Then the Jacobian $\partial(u, v) / \partial(x, y)$ does not vanish at the origin.

Proof. Suppose, without loss of generality, that the power series for $u$ and $v$ in $x$ and $y$ start with non-vanishing terms of $i$ th and $k$ th degree, respectively, and that furthermore $i \leqq k, i>0$. Then the terms of $i$ th degree in $u(x, y)$ form a harmonic function, because this is trivial for $i=1$, and for $i>1$ the only terms in the development of the left hand of (1) of degree $i-2$ are furnished by those of $\partial^{2} u / \partial x^{2}+\partial^{2} u / \partial y^{2}$. Introduce $V(x, y)=v(x, y)-F(u)$, where $F$ is analytic in $u$ and $F(0)=0$. Instead of (1) and (2) we find

$$
\begin{gathered}
\frac{\partial^{2} u}{\partial x^{2}}+\frac{\partial^{2} u}{\partial y^{2}}+\left[a(u, V+F(u))+F^{\prime}(u) b(u, V+F(u))\right. \\
\left.+F^{\prime 2}(u) c(u, V+F(u))\right]\left[\left(\frac{\partial u}{\partial x}\right)^{2}+\left(\frac{\partial u}{\partial y}\right)^{2}\right]+\cdots=0, \\
\frac{\partial^{2} V}{\partial x^{2}}+\frac{\partial^{2} V}{\partial y^{2}}+F^{\prime}(u)\left(\frac{\partial^{2} u}{\partial x^{2}}+\frac{\partial^{2} u}{\partial y^{2}}\right) \\
+\left[A(u, V+F(u))+F^{\prime}(u) B(u, V+F)+F^{\prime 2}(u) C(u, V+F)\right. \\
\left.+F^{\prime \prime}(u)\right]\left[\left(\frac{\partial u}{\partial x}\right)^{2}+\left(\frac{\partial u}{\partial y}\right)^{2}\right]+\cdots=0,
\end{gathered}
$$

and, by substitution of (3) in (4),

$$
\begin{aligned}
\frac{\partial^{2} V}{\partial x^{2}}+\frac{\partial^{2} V}{\partial y^{2}}+ & {\left[A(u, V+F)+F^{\prime} B+F^{\prime 2} C+F^{\prime \prime}-F^{\prime} a\right.} \\
& \left.-F^{\prime 2} b-F^{\prime 3} c\right]\left[\left(\frac{\partial u}{\partial x}\right)^{2}+\left(\frac{\partial u}{\partial y}\right)^{2}\right]+\cdots=0
\end{aligned}
$$

where the omitted terms are linear in

$$
\frac{\partial u}{\partial x} \frac{\partial V}{\partial x}+\frac{\partial u}{\partial y} \frac{\partial V}{\partial y},\left(\frac{\partial V}{\partial x}\right)^{2}+\left(\frac{\partial V}{\partial y}\right), \text { and } \frac{\partial(u, V)}{\partial(x, y)} .
$$

Now notice that $V$ vanishes for $x=y=0$ and that in its development the lowest degree $K$ of non-vanishing terms still is $\geqq i$.

Let us distinguish two cases, $k>i$ and $k=i$. For $k>i$, we con- 
struct a function $F(u)$, solution of the ordinary differential equation of second order

$$
\begin{aligned}
A(u, F(u))+F^{\prime}(u) & B(u, F(u)) \\
+ & F^{\prime 2} C-F^{\prime} a-F^{\prime 2} b-F^{\prime 3} c+F^{\prime \prime}=0,
\end{aligned}
$$

vanishing with its first derivative for $u=0$. We have $k>i$, as $F(u)$, considered as function of $x$ and $y$ starts with terms of degree $\geqq 2 i$. Equation (5) shows now a coefficient of $(\partial u / \partial x)^{2}+(\partial u / \partial y)^{2}$ whose development in $u$ and $V$ has all terms divisible by $V$. Thus the $x, y$ series for the left hand of (5) obtains all terms of degree $K-2$ from the expression $\partial^{2} V / \partial x^{2}+\partial^{2} V / \partial y^{2}$, whence we conclude that in $V$ the terms of lowest order $K$ form a harmonic function. As in the proof of Theorem 1 , we conclude that the Jacobian $\partial(u, V) / \partial(x, y)$, which equals $\partial(u, v) / \partial(x, y)$, would assume both positive and negative values in every neighborhood of the origin, which leads to a contradiction. Thus $i<k$ is impossible.

In the case $i=k$ we again choose $F(u)$ as solution of (6), vanishing for $u=0$, but we try to determine $F^{\prime}(u)$ in such a way that $k$ results $>i$. If this were possible, we could apply the same argument as at the end of the last paragraph, and find the same contradiction. Hence, should $i=k=1$, we would have the desired inequality

$$
\frac{\partial(u, V)}{\partial(x, y)}=\frac{\partial(u, v)}{\partial(x, y)} \neq 0
$$

at the origin. If, however, $i=k>1$, we may determine the linear term of $F(u)$ such that for polar coordinates in the $x y$ plane and suitable determination of $\theta=0$ we have

$$
\begin{aligned}
& u=\text { const. } r^{k} \cos k \theta+r^{k+1}(\cdots), \\
& V=\text { const. } r^{k} \sin k \theta+r^{k+1}(\cdots) .
\end{aligned}
$$

This leads, by the same reasoning as in the proof of Theorem 1 , to the conclusion that the index of the vector field $(u, V)$ at the origin is $k \neq 1$. But together with the functions $u(x, y), v(x, y)$, the functions $u(x, y), V(x, y)=v(x, y)-F(u)$ also establish a one-to-one mapping of the $x y$ plane, because the correspondence between the $u v$ plane and the $u V$ plane evidently is one-to-one. Hence the index ought to be \pm 1 , which completes our proof.

The University of California 